\title{
Response Surface Analysis for Sewage Wastewater Treatment Using Natural Coagulants
}

\author{
Arlene Abuda Joaquin ${ }^{1 *}$, Gnanasundaram Nirmala ${ }^{1}$, Panchanathan Kanakasabai ${ }^{2}$ \\ ${ }^{1}$ School of Chemical Engineering, Vellore Institute of Technology, Vellore, 632014, India \\ ${ }^{2}$ Chemical Engineering Section, Salalah College of Technology, Salalah 211, Oman
}

Received: 29 February 2020

Accepted: 14 April 2020

\begin{abstract}
The studies on utilization of natural coagulants prepared from seeds of Citrullus lanatus (watermelon) and Cucumis melo (cantaloupe) provide insight on sewage wastewater treatment. The results were statistically analyzed by response surface methodology (RSM) based central composite design (CCD), and supported with results from Fourier Transform Infrared (FTIR) spectroscopy for functional groups present in coagulants. The batch coagulation studies were performed varying process parameters such as $\mathrm{pH}(5-7)$ and coagulant dosage $(50-150 \mathrm{mg} / \mathrm{L})$ at constant flocculant dosage of $10 \mathrm{mg} / \mathrm{L}$ using jar test. In jar test, coagulation was carried out with fast mixing (Mixing at $150 \mathrm{rpm}$ for $1 \mathrm{~min}$ ) followed by slow mixing (Mixing at $50 \mathrm{rpm}$ for $30 \mathrm{~min}$ ), and the final mixture was allowed to settle for $1 \mathrm{~h}$ at the temperature of $30 \pm 2^{\circ} \mathrm{C}$. Design-Expert ${ }^{\circledR}$ version 12 software was used to optimize $\mathrm{pH}$ and coagulant dosage for effective sewage wastewater treatment using natural coagulants with the objectives to maximize percentage reduction in turbidity, biological/biochemical oxygen demand (BOD), total suspended solids (TSS), and chemical oxygen demand (COD). The RSM results reveal that the optimum C. lanatus coagulant dosage of $72.3 \mathrm{mg} / \mathrm{L}$ at $\mathrm{pH} 5$ achieved maximum efficacy removal of TSS and BOD by $92.8 \%$ and $92.1 \%$ respectively. The FTIR results show that the coagulants from plant seeds contain almost similar functional groups. Thus, it can be concluded that the coagulant prepared from C. lanatus would be more effective for treatment of sewage wastewater.
\end{abstract}

Keywords: coagulation-flocculation, sewage wastewater, natural coagulant, response surface methodology, FTIR analysis

\section{Introduction}

Nowadays, the treatment and disposal of sewage wastewater is gaining significance in order to protect the quality of terrestrial and aquatic life [1]. Discharging sewage wastewater without proper treatment contributes

*e-mail: joaquin_arlene@yahoo.com to pollution of the water resources and ecosystem [2]. The largest source of water pollution in terms of volume discharged is the sewage wastewater [3]. Sewage wastewater, the water generated from domestic, commercial and public buildings, is composed of bodily wastes (primarily feces and urine) along with the water utilized for toilet flushings, washing utensils, laundry preparation, and cleaning of other materials [4]. The wastewater can be destructive to public health because it contains high TSS, dissolved organic and 
inorganic matter, biological substances and toxic materials [5].

The disposal of wastewater to groundwater or surface water without proper treatment causes risks to human health and environment. Therefore, it is important to identify the appropriate method of treating the wastewater for the welfare of the people and the community with maximum performance and low-cost $[5,6]$. The efficiency of water and wastewater treatment is influenced by the number of stages. The three stages of wastewater treatment include primary, secondary and tertiary. The treatment methods involve water clarification processes such as coagulation, flocculation, sedimentation, disinfection, etc. [7]. One of the principal techniques for wastewater treatment is coagulation and flocculation, used mostly for the treatment of domestic, commercial and industrial wastewater in the world [68]. Coagulation is a process to destabilize charged particles to form a gelatinous mass to settle or to be trapped in the filter. On the other hand, flocculation is done by gentle mixing or agitation so that the particles agglomerate into floc [8].

The coagulation-flocculation process is affected by the type of coagulant, $\mathrm{pH}$, coagulant dosage, flocculant dosage, mixing speed and time for fast and slow mixing in jar test, and the characteristics of wastewater [9]. Moreover, coagulation-flocculation process has been widely employed for potable water and wastewater treatment as a reliable and convenient technique to overcome the forces of stabilizing suspended particles and facilitating the collection and formation of flocs [10-11]. There are different types of coagulants such as natural/organic, inorganic and polymeric materials. Chemical coagulants such as alum [3, 12-14], ferric chloride [12, 14], polyaluminum chloride [13, 15], hydrated lime [3], and calcium carbonate [16] have been used in wastewater treatment to remove pollutants. Most municipal wastewater treatment plants use inorganic polymeric coagulants for turbidity and suspended solids removal [13, 15]. However, the most widely used coagulants for water and wastewater treatment is aluminium sulfate (alum) due to its effectiveness and low-cost although it has been found to cause some health effects to human and create problems to the environment upon long-term usage [3, 14].

On the other hand, biomass based organic polymeric flocculants prepared from Moringa oleifera seeds [17-19], nirmali (Strychnos potatorum) seeds [20-21], tannin [22] and cactus [22] have been the interest of many researchers as the viable alternative choice and tested over the years for different types of water and wastewater treatment due to its efficiency to flocculate with less dosage, high biodegradability, cost effective, abundant availability, eco-friendliness, production of biodegradable sludge, and not likely to produce extreme $\mathrm{pH}[23,24]$. The natural coagulants are generally produced from plant seeds which contain carboxyl, hydroxyl and amino groups that have significant affinity towards various pollutants from their aqueous solutions that enables the coagulation to work effectively [25-27]. In the present study, natural coagulants extracted from the seeds of $C$. lanatus and $C$. melo collected in the Dhofar Governorate of Sultanate of Oman was used. The climate and ecological diversity in the country offers an opportunity to grow offseason fruits and vegetables because of the mild winter climate. The farmers have selected and conserved landraces and local cultivars in a dynamic way.

The fruits of watermelon and cantaloupe are known for its high nutritional value and rich in vitamins and minerals. However, seeds from watermelon are known for its natural coagulant properties that removes the turbidity, BOD, TSS, and COD to greater extent [28, 29]. Furthermore, it is also effective to enhance the filtration process during water treatment with antimicrobial agents [30,31]. On the other hand, cantaloupe is one of the major fruits grown in the middle east. The peel of the fruit is naturally enriched with pectin, $\alpha$-cellulose and non-essential amino acids [32, 33]. Furthermore, the seeds of watermelon and cantaloupe is a good replacement for inorganic and synthetic polyelectrolytes in treating water and wastewater since the natural coagulants are effective and cheaper. The functional goups in the seeds are generally characterized through FTIR. The presence of carboxyl, hydroxyl and amino groups in the coagulant accelerates the effectiveness of coagulation for the maximum removal of turbidity and TSS in municipal wastewater.

A statistical experimental design, RSM, is used in the present study for better understanding of the experimental approach. It works by fitting experimental responses to quadratic function [34]. Also, RSM has successfully helped in the modeling and optimization of various experimental designs involving wastewater treatment process, extraction process, food preservation and other discipline of engineering [3, 15, 26, 32]. The stages involved in the RSM as an optimization tool include: selection of independent variables and their levels, selection of experimental design, generation and validation of model equation, graphical representation and searching for optimal region [35].

The main aim of the study is to investigate the efficacy of natural coagulants (C. lanatus and C. melo) for treatment of sewage wastewater using coagulationflocculation process for the reduction of turbidity, BOD, TSS and COD.

\section{Materials and Methods}

\section{Preparation of Natural Coagulants from C. lanatus (watermelon) and C. melo (cantaloupe) Seeds}

The fresh fruits of watermelon and cantaloupe, grown locally, were procured from the local commercial market in Salalah, Oman. The natural plant seeds were collected from fresh fruits. The seeds were washed several times with double distilled water and 
dried in the hot air oven at $70^{\circ} \mathrm{C}$ to drying. The seeds were sorted to remove debris, shelled and milled to obtain fine powder. The powdered seeds were extracted by means of Soxhlet extraction for about 6 hours using hexane as solvent to obtain a seed cake without oil [36]. The presence of oil in seed could reduce the efficiency of the coagulant. The cake seed is thoroughly washed with double distilled water in order to remove the excess n-hexane, and finally dried in oven at $60^{\circ} \mathrm{C}$ until constant weight and screened through 255 microns and stored in air-tight containers for use as natural powder coagulant. The stock solution of natural coagulant was prepared by adding $1 \mathrm{~g}$ of processed seed cake in $100 \mathrm{~mL}$ of double distilled water.

\section{Jar Test Method}

The most widely applied technique in the sewage treatment plant is coagulation-flocculation due to its effectiveness [3, 37]. The study was performed using the jar test apparatus (Armfield Company, UK) for the evaluation and optimization of coagulationflocculation. The jar test apparatus has stirrers with six blades each rotates with independent variable speed motors at a stirring speed of 0 to $200 \mathrm{rpm}$. The wastewater sample was well-shaken for the re-suspension of possible settling solids and the aliquot volume of sample transferred to the jar test beakers. The coagulant dosage (50-150 mg/L) and $\mathrm{pH}(5-7)$ were the input variables. However, turbidity, BOD, TSS and COD were the dependent variables for coagulationflocculation performance. The $\mathrm{pH}$ of the wastewater sample was adjusted to the desired $\mathrm{pH}$ value of (5.0-7.0) by adding $(1 \mathrm{~N} \mathrm{HCl})$ or $(1 \mathrm{~N} \mathrm{NaOH})$ solution. The experimental process consists of 3 steps: fast mixing at $150 \mathrm{rpm}$ for $1 \mathrm{~min}$ followed by slow mixing for $30 \mathrm{~min}$ at $50 \mathrm{rpm}$ and finally settled for $1 \mathrm{~h}$ at the temperature of $30 \pm 2^{\circ} \mathrm{C}$. After the settling period time, the supernatant was withdrawn and transferred to the flask for subsequesnt analysis and the sludge (dense floc) was discarded. The characterization of supernatant was performed in quadrapulate and the mean value was taken as response, according to the standard operating procedures [38]. The coefficient of variance was found to be within $5 \%$.

\section{Experimental Design and Process Optimization Using RSM}

Design-Expert ${ }^{\circledR}$ version 12 software from Statease Inc., USA was used in the present study for the statistical design of experiments and analysis of data. central composite design (CCD) and Box-Behnken design (BBD) are the two statistical experimental designs in RSM. CCD was selected in the present study to identify the optimal region for the factors affecting the performance of coagulation-flocculation process due to its efficiency [39].
The two most important operating variables in the study were coagulant dosage and $\mathrm{pH}$. The coded values for $\mathrm{pH}$ (A) and coagulant dosage (B) were set at levels: pH $(-1 \leftrightarrow 5.00)$ low, $(+1 \leftrightarrow 7.00)$ high and coagulant dosage $(-1 \leftrightarrow 50.00)$ low, $(+1 \leftrightarrow 150.00)$ high. The response factors considered were percentage reduction in turbidity, TSS, BOD and COD. The number of trials performed in $\mathrm{CCD}$ is $2^{\mathrm{f}}+2 \mathrm{f}+\mathrm{CP}$ where $\mathrm{f}$ is the number of factors or variables and $\mathrm{CP}$ is the number of center points [40]. Trials performed as shown in Table 1 were thirteen (13) with 5 centre points for 2 variables as per the CCD matrix. The experimental data fitted to the quadratic equation was used to study the effect of factors on the response as represented in Equation (1) and model coefficients were evaluated using Equation (2). The coefficients in Equation (1) were analyzed for checking the similarity between experimental and predicted values based on the $p$-value with a $95 \%$ confidence level. The significance of the model was tested using analysis of variance (ANOVA). Regression coefficients $\mathrm{R}^{2}$, adjusted $\mathrm{R}^{2}$ and predicted $\mathrm{R}^{2}$ values were assessed to find the fit between experimental and predicted values. The value between 0.9 and 1 reveals the best of fit between experimental and predicted values.

$\mathrm{Y}=\beta_{0}+\sum_{i=1}^{n} \beta_{i} X_{i}+\sum_{i=1}^{n} \beta_{i i} X_{i}^{2}+\sum_{i=1}^{n-1} \sum_{j=i+1}^{n} \beta_{i j} X_{i} X_{j}+\varepsilon$

$$
\beta=\left(X^{T} X\right)^{-1}\left(X^{T} Y\right)
$$

...where: $\beta_{o}$ is an intercept; $\beta_{i}, \beta_{i i}, \beta_{i j}$ are linear, squared and interaction coefficients respectively, $\mathrm{Y}$ is the response; $X_{i}$ and $X_{j}$ are independent factors; and, $\varepsilon$ is a random error.

\section{FTIR Analysis}

Fourier Transform Infrared (FTIR) spectrophotometer was used to detect functional groups. The samples $C$. lanatus and $C$. melo were grounded and then compressed and formed into pellets. The pellets formed were analyzed covering a frequency range of 4000-400 $\mathrm{cm}^{-1}$ with a resolution of $4 \mathrm{~cm}^{-1}$ at room temperature [41].

\section{Analytical Methods}

The sewage wastewater sample was collected from Salalah Sanitary Drainage Services Company SAOC (SSDS) water reclamation plant where the flow rate is $35,000-40,000 \mathrm{~m}^{3} /$ day [42]. The sewage wastewater sample collected in an air-tight glass container was characterized for physiochemical parameters such as turbidity, TSS, BOD and COD. All experiments were performed to determine the physiochemical parameters in quadrapulate to ensure the results with minimum error. The $\mathrm{pH}$ of the solution was measured using a $\mathrm{pH}$ Meter (Hanna HI2211-02) with regular calibrations of 4.01, 7.0 and 10.0 standard $\mathrm{pH}$ buffer solutions (Thermo 
Scientific Orion). The turbidity was measured using the ISO Turbidity Portable Meter (HI98713-01). However, the BOD was measured using 5-day DO test as per the APHA standard method and COD was measured using the dichromate open reflux method as per standard methods [43]. The TSS values were measured using gravimetric method.

The percentage removal efficiencies of turbidity, TSS, COD and BOD was determined according to Equation (3):

$$
\begin{gathered}
\% \text { Removal }=(\text { Cinfluent }- \text { Ceffluent }) \div(\text { Cinfluent }) \\
\times 100
\end{gathered}
$$

...where: Cinfluent and Ceffluent are the concentrations of TSS, COD and BOD in the influent and effluent respectively.

\section{Results and Discussion}

\section{Characteristics of Sewage Wastewater Collected}

The sewage wastewater was characterized and $\mathrm{pH}$, turbidity, TSS, BOD and COD were found to be $7.25,225 \mathrm{mg} / \mathrm{L}, 500 \mathrm{mg} / \mathrm{L}, 60 \mathrm{mg} / \mathrm{L}$ and $575 \mathrm{mg} / \mathrm{L}$ respectively.

\section{Assessment of Statistical Modeling and Optimization through RSM}

Tables 1 and 2 show the results of the CCD matrix designed for input variables with percentage reduction in turbidity, COD, TSS and BOD using C. lanatus and $C$. melo as natural coagulants. The independent variables (inputs) used in this experiment were $\mathrm{pH}$ (5-7) and coagulant dosage (50-150 $\mathrm{mg} / \mathrm{L})$. The reduction percentage in turbidity, TSS, BOD and COD were the responses or dependent variables. Optimization was carried out for thirteen experiments to compare the corresponding experimental and predicted results for both coagulants for removal efficiencies of responses as given in Table 3. From the results obtained in the experiments, quadratic model was recommended to represent the performance of coagulation-flocculation process for each coagulant used in the experiments. The $\mathrm{pH}$ and dosage are both significant terms to yield higher percentage removal of TSS [43]. The TSS response surface indicates $92.8 \%$ removal efficiency, which agrees with findings using conventional coagulants found to be the same in C. lanatus natural coagulant.

Tables 4 and 5 show the analysis of variance (ANOVA) results of the quadratic models. The results were assessed with various descriptive statistics such as; $p$ value, lack of fit test, coefficients of determination $R^{2}$ and adequate precision. The $p$-value is used to estimate whether the $\mathrm{F}$ value is large enough to indicate statistical significance [44]. The higher Fishers's $F$-test values and low of $p$-values $(<0.05)$ imply that the quadratic model terms are significant [45]. However, $p$-values $\geq 0.05$ indicate the insignificant model terms. Thus, results in quadratic models predicted in $C$. lanatus are higher in $F$-test and $p$-value less than 0.05 (Table 4). For $C$. melo $\mathrm{F}$-value is lower than $C$. lanatus with $p$-value less than 0.05 (Table 5) which means that both results were

Table 1. CCD matrix for reduction of Turbidity, TSS, BOD and COD by coagulation using citrullus lanatus coagulant.

\begin{tabular}{|c|c|c|c|c|c|c|c|}
\hline & Factor 1 & Factor 2 & Response 1 & Response 2 & Response 3 & Response 4 \\
\hline Std & Run & A:pH & $\begin{array}{c}\text { B:Coagulant } \\
\text { dosage }\end{array}$ & $\begin{array}{c}\text { Reduction in } \\
\text { Turbidity }\end{array}$ & $\begin{array}{c}\text { Reduction in } \\
\text { TSS }\end{array}$ & $\begin{array}{c}\text { Reduction in } \\
\text { BOD }\end{array}$ & $\begin{array}{c}\text { Reduction in } \\
\text { COD }\end{array}$ \\
\hline 11 & 1 & 6 & 100 & 37.6 & 87.7 & 95.5 & 71.4 \\
\hline 5 & 2 & 5 & 100 & 37.6 & 92.2 & 97.7 & 71.4 \\
\hline 7 & 3 & 6 & 50 & 96.6 & 86.7 & 76.1 & 10 \\
\hline 13 & 4 & 6 & 100 & 37.6 & 87.7 & 95.5 & 71.4 \\
\hline 8 & 5 & 6 & 150 & 35.6 & 76.7 & 95.5 & 78.6 \\
\hline 4 & 6 & 7 & 150 & 40 & 77.2 & 88.1 & 71.4 \\
\hline 1 & 7 & 5 & 50 & 95.5 & 93.3 & 86.4 & 14.3 \\
\hline 6 & 8 & 7 & 100 & 34.1 & 87.7 & 85.2 & 71.4 \\
\hline 9 & 9 & 6 & 100 & 37.6 & 87.7 & 95.5 & 71.4 \\
\hline 2 & 10 & 7 & 50 & 92.8 & 85.5 & 50 & 34.3 \\
\hline 10 & 11 & 6 & 100 & 37.6 & 87.7 & 95.5 & 71.4 \\
\hline 12 & 12 & 6 & 100 & 37.6 & 87.7 & 95.5 & 71.4 \\
\hline 3 & 13 & 5 & 150 & 36.5 & 79.5 & 96.6 & 81.4 \\
\hline
\end{tabular}


Table 2. CCD matrix for reduction of Turbidity, TSS, BOD and COD by coagulation using cucumis melo coagulant.

\begin{tabular}{|c|c|c|c|c|c|c|c|}
\hline & & Factor 1 & Factor 2 & Response 1 & Response 2 & Response 3 & Response 4 \\
\hline Std & Run & A:pH & $\begin{array}{l}\text { B:Coagulant } \\
\text { dosage }\end{array}$ & $\begin{array}{l}\text { Reduction in } \\
\text { Turbidity }\end{array}$ & $\begin{array}{c}\text { Reduction in } \\
\text { TSS }\end{array}$ & $\begin{array}{c}\text { Reduction in } \\
\text { BOD }\end{array}$ & $\begin{array}{l}\text { Reduction in } \\
\text { COD }\end{array}$ \\
\hline & & & $\mathrm{mg} / \mathrm{L}$ & $\%$ & $\%$ & $\%$ & $\%$ \\
\hline 11 & 1 & 6 & 100 & 53.2 & 91.1 & 95.4 & 28.6 \\
\hline 5 & 2 & 5 & 100 & 30.1 & 88.9 & 88.6 & 11.4 \\
\hline 7 & 3 & 6 & 50 & 59.5 & 87.7 & 39.8 & 88.6 \\
\hline 13 & 4 & 6 & 100 & 53.2 & 91.1 & 95.4 & 28.6 \\
\hline 8 & 5 & 6 & 150 & 46.7 & 89.4 & 96 & 50 \\
\hline 4 & 6 & 7 & 150 & 69.1 & 77.7 & 93.3 & 69.9 \\
\hline 1 & 7 & 5 & 50 & 41.8 & 64.4 & 44 & 22.9 \\
\hline 6 & 8 & 7 & 100 & 88.2 & 87.7 & 94.3 & 57.1 \\
\hline 9 & 9 & 6 & 100 & 53.2 & 91.1 & 95.4 & 28.6 \\
\hline 2 & 10 & 7 & 50 & 68.1 & 87.7 & 65.9 & 71.4 \\
\hline 10 & 11 & 6 & 100 & 53.2 & 91.1 & 95.4 & 28.6 \\
\hline 12 & 12 & 6 & 100 & 53.2 & 91.1 & 95.4 & 28.6 \\
\hline 3 & 13 & 5 & 150 & 32.1 & 88.4 & 92 & 48.6 \\
\hline
\end{tabular}

significant. Therefore, the model developed in the study was statistically significant in $( \pm 5 \%)$.

The adequate precision ratios obtained as shown in Table 6 (C. lanatus) and Table 7 (C. melo) were greater than 4 , indicating that the adequate signal for all models could be used to navigate the design space. The lack of fit test describes the variation of data around the fitted model. If the quadratic models fit the data, the lack-of-fit test will be insignificant [46]. Table 4 and 5 indicate that the lack-of-fit values in all models are insignificant. The ANOVA of regression model $\left(R^{2}\right)$ greater than 0.98 values indicates the aptness of the model. $R^{2}$ values in $C$. lanatus (Table 6) are all greater than 0.81 while in $C$. melo (Table 7) all values were reduced. Moreover, $R^{2}$ values close to 1 denote a satisfactory adjustment of the quadratic models to the experimental data. This phenomenon justified the high treatment efficiency removal in $C$. lanatus rather than C. melo coagulant.

The 3-D response surface plots of the regression equation (1) are presented in Figs 1 and 2. The effect of natural coagulant dosage and $\mathrm{pH}$ and their interactions on a percentage reduction in turbidity, BOD, TSS, and COD are shown in Figs 1(a-d) and 2 (a-d) for watermelon (C. lanatus) and cantaloupe (C. melo) respectively. The results of the experiments conducted for the effect of $\mathrm{pH}$ and dosage on turbidity and TSS removal are presented in Fig. 1(a-b) and Fig. 2(a-b). Results from Fig. 1(a-b) showed that at $\mathrm{pH} 5$ and coagulant dosage of $50 \mathrm{mg} / \mathrm{L}$, the removal of turbidity and TSS were high at 95.5 and $93.3 \%$ respectively. When coagulant dosage was increased to $100 \mathrm{mg} / \mathrm{L}$, the removal of turbidity and TSS were reduced to 37.6 and $92.2 \%$ respectively. On the other

Table 3. Optimal values for reduction of Turbidity, TSS, BOD and COD by coagulation using Citrullus lantus and Cucumis melo coagulant.

\begin{tabular}{|c|c|c|c|c|c|c|}
\hline \multirow{2}{*}{$\begin{array}{c}\text { Natural } \\
\text { Coagulant }\end{array}$} & $\mathrm{pH}$ & $\begin{array}{c}\text { Natural Coagulant } \\
\text { Dosage }\end{array}$ & \multirow{2}{*}{ \% Turbidity Reduction } & \% TSS Reduction & \% BOD Reduction & \% COD Reduction \\
\cline { 3 - 6 } & $\mathrm{mg} / \mathrm{L}$ & & 66.5 (Experimental) & $\begin{array}{c}92.8 \text { (Experimen- } \\
\text { tal) }\end{array}$ & 92.1 (Experimental) & $\begin{array}{c}42.9 \text { (Experimen- } \\
\text { tal) }\end{array}$ \\
\hline $\begin{array}{c}\text { Citrullus } \\
\text { lanatus }\end{array}$ & 5 & 72.3 & 62.8 (Predicted) & 94.2 (Predicted) & 94.9 (Predicted) & 44.3 (Predicted) \\
\hline \multirow{2}{*}{$\begin{array}{c}\text { Cucumis } \\
\text { Melo }\end{array}$} & 7 & 76.7 & 78.2 (Experimental) & $\begin{array}{c}87.7 \text { (Experimen- } \\
\text { tal) }\end{array}$ & 80.1 (Experimental) & $\begin{array}{c}64.3 \text { (Experimen- } \\
\text { tal) }\end{array}$ \\
\cline { 3 - 6 } & & 77.0 (Predcted) & 89.6 (Predicted) & 86.9 (Predicted) & 56.9 (Predicted) \\
\hline
\end{tabular}




\begin{tabular}{|c|c|c|c|c|c|c|c|c|c|c|c|}
\hline \multirow{4}{*}{ 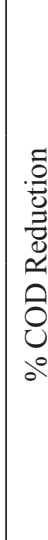 } & 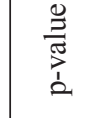 & $\begin{array}{l}\overline{8} \\
\dot{0} \\
\dot{0} \\
\text { v }\end{array}$ & 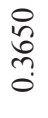 & $\begin{array}{l}\overline{8} \\
\dot{8} \\
\dot{0} \\
\mathrm{v}\end{array}$ & $\begin{array}{l}\tilde{O} \\
\text { oे } \\
0\end{array}$ & 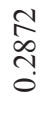 & $\begin{array}{l}\overrightarrow{8} \\
\dot{8} \\
\dot{0} \\
v\end{array}$ & & & & \\
\hline & 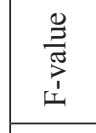 & 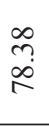 & 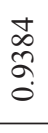 & 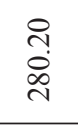 & $\begin{array}{l}\hat{\sigma} \\
\stackrel{+}{J}\end{array}$ & $\stackrel{m}{?}$ & $\begin{array}{l}\bar{\alpha} \\
\dot{\alpha}\end{array}$ & & & & \\
\hline & 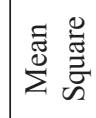 & 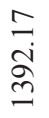 & $\begin{array}{l}\hat{\sigma} \\
\stackrel{0}{0}\end{array}$ & $\begin{array}{l}\vec{d} \\
\dot{0} \\
\stackrel{a}{G}\end{array}$ & $\begin{array}{l}8 \\
\ddot{3} \\
\text { in }\end{array}$ & 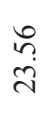 & 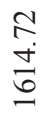 & $\stackrel{0}{\stackrel{0}{\beth}}$ & $\frac{\text { J }}{\dot{\forall}}$ & 音 & \\
\hline & 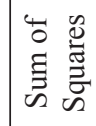 & $\begin{array}{l}\hat{\infty} \\
\dot{0} \\
8 \\
0\end{array}$ & $\begin{array}{l}\hat{6} \\
\stackrel{0}{0}\end{array}$ & $\begin{array}{l}\text { ț } \\
\stackrel{0}{\circ} \\
\stackrel{\sigma}{\sigma}\end{array}$ & 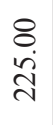 & 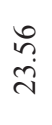 & 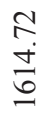 & 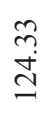 & $\stackrel{\overbrace{}}{\stackrel{\overbrace{}}{ \pm}}$ & ڤे & $\begin{array}{l}\stackrel{2}{\vec{\alpha}} \\
\stackrel{\infty}{2}\end{array}$ \\
\hline \multirow{4}{*}{ 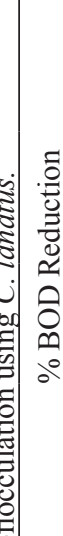 } & 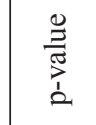 & $\begin{array}{l}\overline{8} \\
\dot{0} \\
\text { v }\end{array}$ & $\begin{array}{l}\overline{8} \\
\dot{0} \\
\mathrm{v}\end{array}$ & $\begin{array}{l}\bar{\Xi} \\
\dot{8} \\
\dot{v}\end{array}$ & 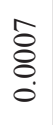 & $\begin{array}{l}\stackrel{n}{g} \\
\stackrel{0}{0} \\
0\end{array}$ & ठิ & & & & \\
\hline & 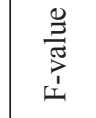 & $\begin{array}{l}\stackrel{q}{+} \\
\infty \\
0\end{array}$ & $\stackrel{f}{\stackrel{f}{+}}$ & 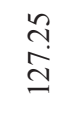 & $\begin{array}{c}\text { I } \\
\text { in }\end{array}$ & $\stackrel{\text { fo }}{\stackrel{0}{0}}$ & $\begin{array}{l}\infty \\
\infty \\
\stackrel{q}{q}\end{array}$ & & & & \\
\hline & 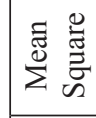 & $\begin{array}{l}\vec{J} \\
\stackrel{0}{\sigma} \\
\forall\end{array}$ & $\begin{array}{l}m \\
\stackrel{9}{a} \\
\dot{a} \\
\text { in }\end{array}$ & $\begin{array}{l}\infty \\
\infty \\
\infty \\
0 \\
i\end{array}$ & $\begin{array}{l}8 \\
+ \\
0\end{array}$ & $\begin{array}{l}\hat{\sigma} \\
\text { î }\end{array}$ & $\begin{array}{l}\text { テे } \\
\text { ๙े }\end{array}$ & $\underset{6}{8}$ & $\begin{array}{l}\overrightarrow{0} \\
\dot{J}\end{array}$ & $\begin{array}{l}8 \\
\vdots \\
0 \\
0\end{array}$ & \\
\hline & 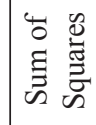 & $\begin{array}{l}\infty \\
\stackrel{\infty}{\infty} \\
\hat{\tilde{N}}\end{array}$ & $\stackrel{m}{\stackrel{9}{+}}$ & $\begin{array}{l}\infty \\
\infty \\
\infty \\
\infty\end{array}$ & $\begin{array}{l}8 \\
+ \\
0\end{array}$ & $\begin{array}{l}\hat{\sigma} \\
\text { तु }\end{array}$ & $\begin{array}{l}\text { ने. } \\
\text { बे }\end{array}$ & $\begin{array}{l}\text { Õ } \\
\text { I }\end{array}$ & $\begin{array}{l}\text { ठै. } \\
\text { fं }\end{array}$ & : & 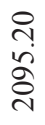 \\
\hline \multirow{4}{*}{ 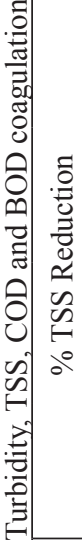 } & 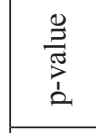 & $\begin{array}{l}\vec{\Xi} \\
\dot{0} \\
\dot{0} \\
\mathrm{v} \\
\end{array}$ & $\begin{array}{l}\overrightarrow{8} \\
\dot{0} \\
\dot{0} \\
\mathrm{v}\end{array}$ & $\begin{array}{l}\overrightarrow{0} \\
\dot{0} \\
0 \\
\mathrm{v}\end{array}$ & $\begin{array}{l}\vec{\Xi} \\
\dot{0} \\
\dot{0} \\
\mathrm{v}\end{array}$ & $\begin{array}{l}\vec{\Xi} \\
\dot{0} \\
\dot{0} \\
\mathrm{v}\end{array}$ & $\begin{array}{l}\vec{\Xi} \\
\dot{0} \\
\dot{0} \\
\mathrm{v}\end{array}$ & & & & \\
\hline & 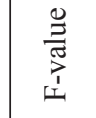 & $\begin{array}{c}n \\
\cdots \\
n\end{array}$ & $\begin{array}{l}\text { ț } \\
\text { ठ̀ं } \\
\text { ஸे }\end{array}$ & $\begin{array}{l}\hat{A} \\
\dot{y} \\
\dot{y} \\
\text { in }\end{array}$ & $\begin{array}{l}\Xi \\
\stackrel{\vec{I}}{\Xi}\end{array}$ & 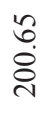 & $\begin{array}{l}\frac{n}{\mathfrak{d}} \\
\frac{a}{g}\end{array}$ & & & & \\
\hline & 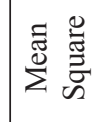 & $\stackrel{\circ}{\stackrel{0}{0}}$ & $\begin{array}{l}n \\
\hat{n} \\
m\end{array}$ & $\stackrel{+}{\stackrel{+}{I}}$ & $\begin{array}{l}\stackrel{n}{r} \\
r\end{array}$ & $\begin{array}{l}\stackrel{+}{w} \\
\stackrel{n}{n}\end{array}$ & $\begin{array}{l}\hat{6} \\
\stackrel{8}{8}\end{array}$ & 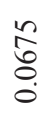 & $\frac{0}{5}$ & ठ & \\
\hline & 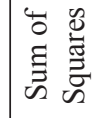 & $\begin{array}{c}\infty \\
\stackrel{\infty}{n} \\
m \\
m\end{array}$ & $\begin{array}{l}\hat{n} \\
\stackrel{n}{n} \\
n\end{array}$ & $\stackrel{\stackrel{+}{ }}{\stackrel{ }{I}}$ & 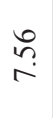 & 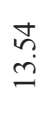 & $\begin{array}{l}\hat{b} \\
\dot{8}\end{array}$ & 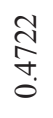 & 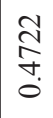 & ठ̊. & $\begin{array}{l}n \\
\hat{n} \\
\dot{m}\end{array}$ \\
\hline \multirow{4}{*}{ 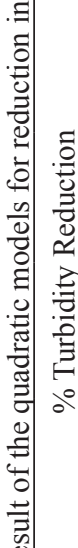 } & 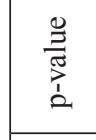 & $\begin{array}{l}\vec{\Xi} \\
\vdots \\
\dot{0} \\
\mathrm{v} \\
\end{array}$ & $\begin{array}{l}\text { ळे } \\
\text { वे }\end{array}$ & $\begin{array}{l}\vec{\Xi} \\
\dot{8} \\
\dot{0} \\
\mathrm{v}\end{array}$ & 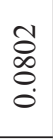 & 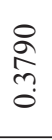 & $\begin{array}{l}\overrightarrow{\dot{a}} \\
\dot{0} \\
\mathrm{v}\end{array}$ & & & & \\
\hline & 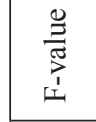 & $\frac{\vec{n}}{\overrightarrow{6}}$ & 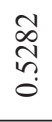 & $\begin{array}{l}\vec{i} \\
\overrightarrow{0} \\
\vec{n}\end{array}$ & $\stackrel{\infty}{\underset{\sim}{*}}$ & \begin{tabular}{l}
\multicolumn{1}{c}{} \\
$\infty$ \\
$\infty$ \\
0 \\
0
\end{tabular} & 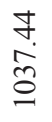 & & & & \\
\hline & 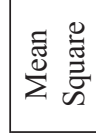 & $\begin{array}{l}\tilde{D} \\
\tilde{f} \\
\stackrel{\sim}{n} \\
-\end{array}$ & ָี & 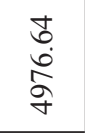 & $\begin{array}{l}\vec{\sigma} \\
\circ\end{array}$ & $\stackrel{\tilde{c}}{\hat{i}}$ & $\begin{array}{l}0 \\
\stackrel{0}{0} \\
\infty \\
\stackrel{\sim}{0}\end{array}$ & 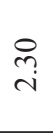 & ñ & 离 & \\
\hline & 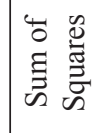 & $\begin{array}{l}\infty \\
0 \\
\stackrel{0}{2} \\
\stackrel{2}{\wedge}\end{array}$ & $\stackrel{\text { తి }}{-}$ & 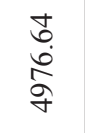 & $\vec{\sigma}$ & $\underset{\mathrm{r}}{\tilde{i}}$ & 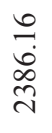 & $\frac{0}{0}$ & $\stackrel{0}{0}$ & 离 & 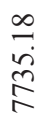 \\
\hline & tz & in & - & - & - & - & - & $r$ & $m$ & $\nabla$ & $\simeq$ \\
\hline & $\exists$ & $\frac{0}{0}$ & 竞 & 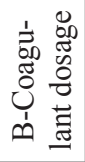 & $\frac{\pi}{4}$ & $\stackrel{z}{<}$ & $\tilde{\emptyset}$ & 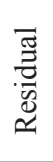 & 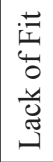 & 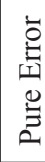 & $\begin{array}{l}\frac{\bar{\pi}}{6} \\
\stackrel{0}{0} \\
\overline{0}\end{array}$ \\
\hline
\end{tabular}

\begin{tabular}{|c|c|c|c|c|c|c|c|c|c|c|c|}
\hline \multirow{4}{*}{ 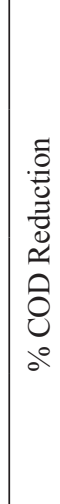 } & 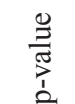 & $\begin{array}{l}\hat{\partial} \\
\dot{0}\end{array}$ & $\begin{array}{l}\overrightarrow{\widehat{\theta}} \\
\dot{0}\end{array}$ & $\begin{array}{l}\infty \\
\stackrel{0}{0} \\
0 \\
0\end{array}$ & $\begin{array}{l}\stackrel{0}{2} \\
\tilde{n} \\
0\end{array}$ & $\begin{array}{l}\bar{J} \\
\stackrel{0}{0}\end{array}$ & $\begin{array}{l}\hat{o} \\
\vdots \\
0\end{array}$ & & & & \\
\hline & 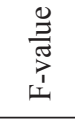 & $\begin{array}{l}n \\
\infty \\
\infty \\
n\end{array}$ & 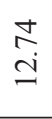 & $\begin{array}{l}\stackrel{\infty}{\circ} \\
\stackrel{+}{0}\end{array}$ & $\stackrel{\bullet}{-}$ & 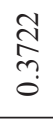 & $\begin{array}{l}\stackrel{\vartheta}{ \pm} \\
\dot{J}\end{array}$ & & & & \\
\hline & 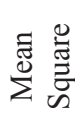 & 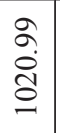 & 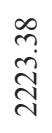 & $\begin{array}{l}\stackrel{0}{n} \\
\stackrel{n}{m}\end{array}$ & $\begin{array}{l}\stackrel{0}{0} \\
\dot{0}\end{array}$ & $\begin{array}{l}\hat{a} \\
\text { ț }\end{array}$ & $\begin{array}{l}\hat{a} \\
\infty \\
\vec{n}\end{array}$ & $\begin{array}{l}\hat{n} \\
\stackrel{+}{ \pm}\end{array}$ & $\begin{array}{l}\stackrel{2}{a} \\
\stackrel{+}{+}\end{array}$ & $\begin{array}{l}8 \\
8 \\
0 \\
0\end{array}$ & \\
\hline & 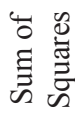 & $\begin{array}{l}\stackrel{+}{\circ} \\
\dot{0} \\
\stackrel{\vec{n}}{n}\end{array}$ & $\begin{array}{l}\infty \\
\stackrel{\infty}{3} \\
\underset{N}{N}\end{array}$ & $\begin{array}{l}\stackrel{0}{ } \\
\stackrel{n}{m}\end{array}$ & 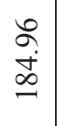 & $\begin{array}{l}\hat{a} \\
\text { ț }\end{array}$ & $\begin{array}{l}\hat{a} \\
\infty \\
\vec{a} \\
\vec{a}\end{array}$ & $\begin{array}{l}\stackrel{\circ}{\circ} \\
\stackrel{\vec{\Xi}}{\Xi}\end{array}$ & $\begin{array}{l}\stackrel{\circ}{\circ} \\
\stackrel{\vec{\lambda}}{\Xi}\end{array}$ & $\begin{array}{l}8 \\
8 \\
0 \\
0\end{array}$ & 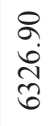 \\
\hline \multirow{4}{*}{ 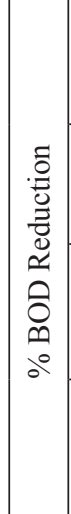 } & 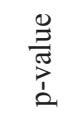 & $\begin{array}{l}\vec{\delta} \\
\dot{0} \\
\dot{0} \\
v\end{array}$ & ڤે & $\begin{array}{l}\vec{\delta} \\
\dot{8} \\
\dot{0} \\
v\end{array}$ & $\begin{array}{l}m \\
\hat{8} \\
0 \\
0\end{array}$ & $\begin{array}{l}8 \\
\stackrel{0}{0} \\
\infty \\
0\end{array}$ & 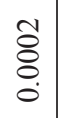 & & & & \\
\hline & 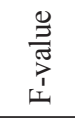 & $\begin{array}{l}: \\
\dot{d} \\
\dot{m}\end{array}$ & $\begin{array}{l}8 \\
\text { in }\end{array}$ & $\begin{array}{l}\bar{\sigma} \\
\dot{\Xi}\end{array}$ & $\begin{array}{l}\infty \\
\infty \\
r\end{array}$ & $\begin{array}{l}\overrightarrow{0} \\
\varnothing \\
0\end{array}$ & $\frac{\tilde{\sigma}}{\bar{n}}$ & & & & \\
\hline & 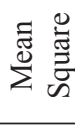 & $\begin{array}{l}\tilde{\sigma} \\
\text { in } \\
\alpha\end{array}$ & $\begin{array}{l}\text { ণิ } \\
\text { ભે }\end{array}$ & 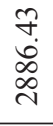 & $\begin{array}{l}\dot{g} \\
\dot{\delta}\end{array}$ & $\stackrel{શ}{i}$ & 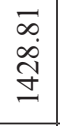 & $\begin{array}{l}\vec{n} \\
\stackrel{\sim}{n}\end{array}$ & 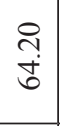 & $\begin{array}{l}8 \\
\vdots \\
\vdots \\
0\end{array}$ & \\
\hline & 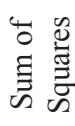 & $\begin{array}{l}\stackrel{0}{-} \\
\stackrel{0}{0} \\
\stackrel{8}{f}\end{array}$ & $\begin{array}{l}\text { సે } \\
\text { ๙ે } \\
-\end{array}$ & 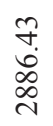 & $\begin{array}{l}\stackrel{0}{0} \\
\stackrel{0}{0}\end{array}$ & $\stackrel{\vartheta}{\stackrel{i}{.}}$ & $\begin{array}{l}\vec{\infty} \\
\infty \\
\stackrel{J}{J} \\
\stackrel{J}{-}\end{array}$ & $\begin{array}{c}\stackrel{8}{ } \\
\stackrel{i}{\Omega} \\
\Omega\end{array}$ & $\begin{array}{l}\stackrel{B}{ } \\
i \\
\sigma \\
-\end{array}$ & $\begin{array}{l}8 \\
8 \\
0 \\
0\end{array}$ & $\begin{array}{l}\stackrel{R}{i} \\
\text { i } \\
\alpha \\
\sigma\end{array}$ \\
\hline & 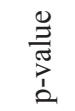 & $\begin{array}{l}\circ \\
\stackrel{0}{\circ} \\
\stackrel{0}{0}\end{array}$ & $\begin{array}{l}\stackrel{2}{\circ} \\
\stackrel{2}{0}\end{array}$ & 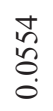 & $\begin{array}{l}n \\
\vdots \\
0 \\
0 \\
0\end{array}$ & $\begin{array}{l}\hat{0} \\
0 \\
0 \\
0\end{array}$ & $\begin{array}{l}\stackrel{1}{0} \\
\stackrel{0}{0} \\
0\end{array}$ & & & & \\
\hline
\end{tabular}

\begin{tabular}{|c|c|c|c|c|c|c|c|c|c|c|}
\hline \multirow[t]{3}{*}{.} & 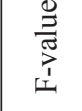 & 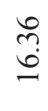 & 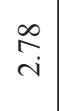 & $\begin{array}{l}\stackrel{i}{n} \\
\text { in }\end{array}$ & $\begin{array}{l}0 \\
\dot{d} \\
\dot{m}\end{array}$ & $\begin{array}{l}\infty \\
\stackrel{\infty}{=}\end{array}$ & $\begin{array}{l}\infty \\
\infty \\
0 \\
0\end{array}$ & & & \\
\hline & 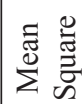 & 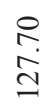 & $\begin{array}{l}: \\
\stackrel{\sim}{\sim} \\
\end{array}$ & $\begin{array}{l}\stackrel{\infty}{\stackrel{+}{\forall}} \\
\stackrel{\nabla}{*}\end{array}$ & 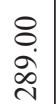 & $\begin{array}{c}\hat{\sigma} \\
\text { à }\end{array}$ & $\begin{array}{l}\infty \\
\infty \\
\dot{0} \\
\infty\end{array}$ & $\stackrel{\triangleright}{\stackrel{\infty}{\sim}}$ & $\begin{array}{l}\overrightarrow{\hat{y}} \\
-\infty\end{array}$ & $\begin{array}{l}8 \\
8 \\
0 \\
0\end{array}$ \\
\hline & 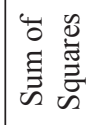 & $\begin{array}{l}\infty \\
\stackrel{\infty}{0} \\
\infty \\
\widehat{0}\end{array}$ & $\begin{array}{l}\stackrel{\bullet}{:} \\
\stackrel{\vec{\lambda}}{1}\end{array}$ & $\stackrel{\infty}{\stackrel{\infty}{\dot{\forall}}}$ & $\begin{array}{l}8 \\
\stackrel{0}{0} \\
\stackrel{0}{1}\end{array}$ & $\begin{array}{l}\text { oे } \\
\text { ì }\end{array}$ & $\begin{array}{l}\infty \\
\infty \\
\dot{\infty}\end{array}$ & $\begin{array}{l}\text { Sర } \\
\stackrel{+}{n}\end{array}$ & $\begin{array}{l}\tilde{\sigma} \\
\dot{\sim} \\
\dot{n}\end{array}$ & $\begin{array}{l}8 \\
\vdots \\
\vdots \\
0\end{array}$ \\
\hline \multirow{4}{*}{ 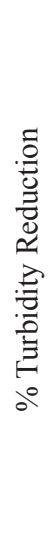 } & 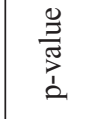 & $\begin{array}{l}\overrightarrow{\widetilde{\delta}} \\
\dot{\delta}\end{array}$ & $\begin{array}{l}\overrightarrow{8} \\
\dot{8} \\
\dot{0}\end{array}$ & \begin{tabular}{l}
$\hat{0}$ \\
\multirow{1}{0}{}
\end{tabular} & $\begin{array}{l}\infty \\
\infty \\
\stackrel{+}{+} \\
\stackrel{0}{0}\end{array}$ & $\begin{array}{l}\stackrel{0}{7} \\
\stackrel{+}{+} \\
0\end{array}$ & 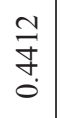 & & & \\
\hline & 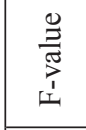 & $\begin{array}{l}\stackrel{0}{\infty} \\
\text { Ij }\end{array}$ & $\begin{array}{l}\vec{n} \\
\tilde{8}\end{array}$ & $\stackrel{9}{9}$ & \begin{tabular}{|l}
$\overrightarrow{\hat{n}}$ \\
$\grave{0}$ \\
$\dot{0}$
\end{tabular} & $\begin{array}{c}0 \\
0 \\
0 \\
n \\
0 \\
0\end{array}$ & $\begin{array}{l}\tilde{0} \\
0 \\
0 \\
0\end{array}$ & & & \\
\hline & 宽 & $\frac{t}{a}$ & 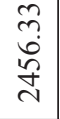 & 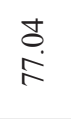 & $\begin{array}{c}\mathcal{C} \\
\infty \\
d \\
d\end{array}$ & 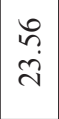 & $\begin{array}{l}n \\
\dot{d} \\
\dot{\sim}\end{array}$ & $\begin{array}{l}\hat{n} \\
\hat{q}\end{array}$ & $\begin{array}{l}\mathbb{N} \\
\dot{d}\end{array}$ & $\begin{array}{l}8 \\
\vdots \\
0 \\
0\end{array}$ \\
\hline & 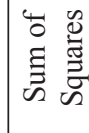 & $\begin{array}{l}\hat{b} \\
\infty \\
0 \\
\hat{d}\end{array}$ & 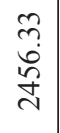 & 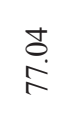 & $\mid \begin{array}{c}\mathfrak{C} \\
\infty \\
\infty \\
\sim \\
\sim\end{array}$ & 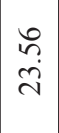 & 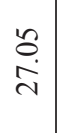 & $\begin{array}{l}0 \\
\stackrel{+}{+} \\
\stackrel{\sim}{\sim}\end{array}$ & $\begin{array}{l}0 \\
\stackrel{+}{+} \\
\infty \\
\stackrel{0}{\sim}\end{array}$ & $\begin{array}{l}8 \\
8 \\
0 \\
0\end{array}$ \\
\hline & 㟧 & in & - & - & - & - & - & $r$ & $m$ & $\nabla$ \\
\hline & 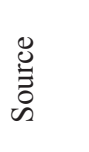 & \begin{tabular}{|l}
$\bar{\nabla}$ \\
$\bar{\delta}$ \\
$\bar{\Sigma}$
\end{tabular} & 兑 & 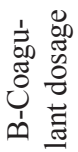 & $\frac{m}{2}$ & $\mathbb{Z}$ & $\tilde{\omega}$ & 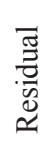 & 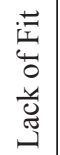 & 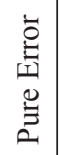 \\
\hline
\end{tabular}



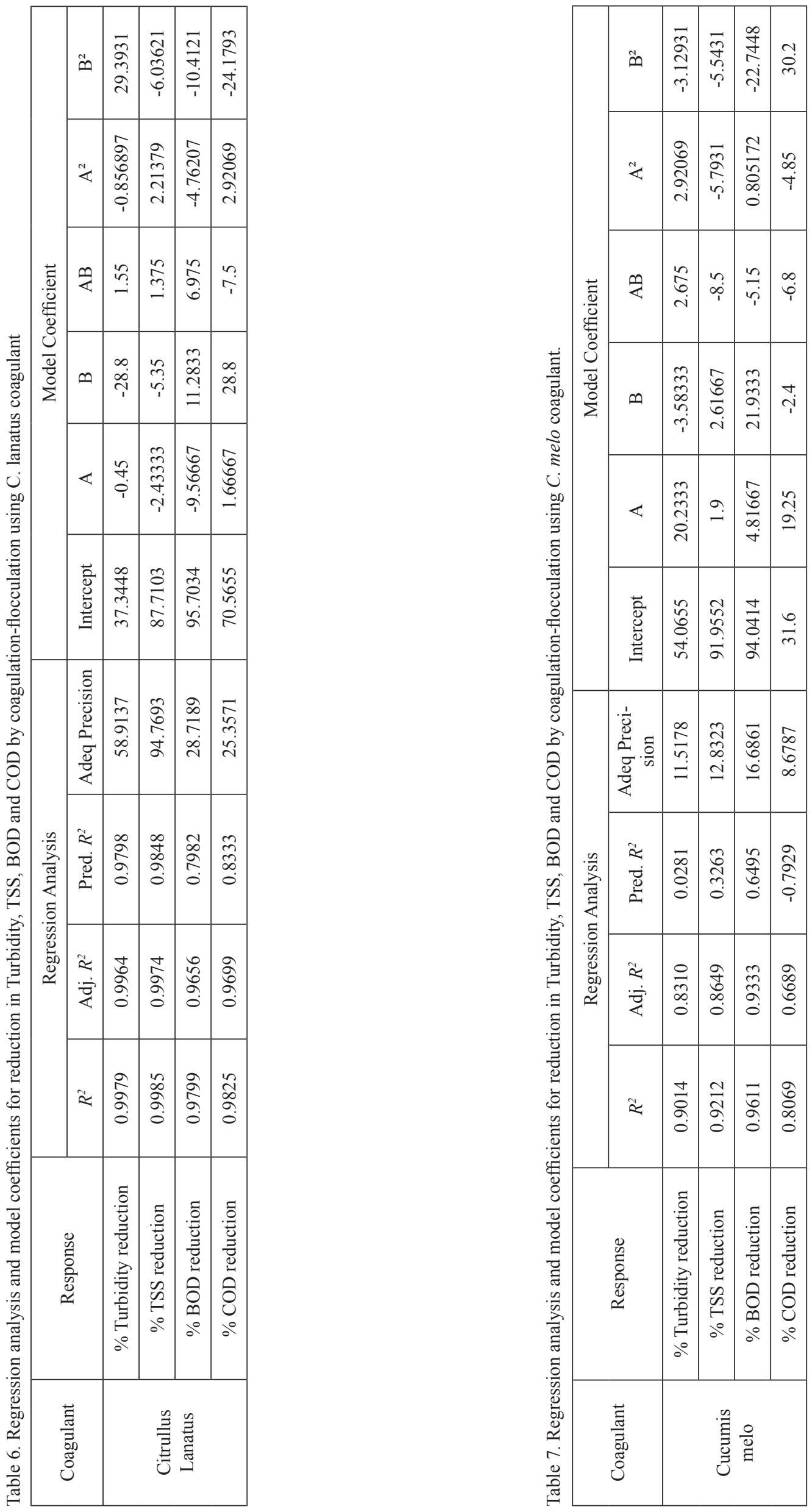
a)

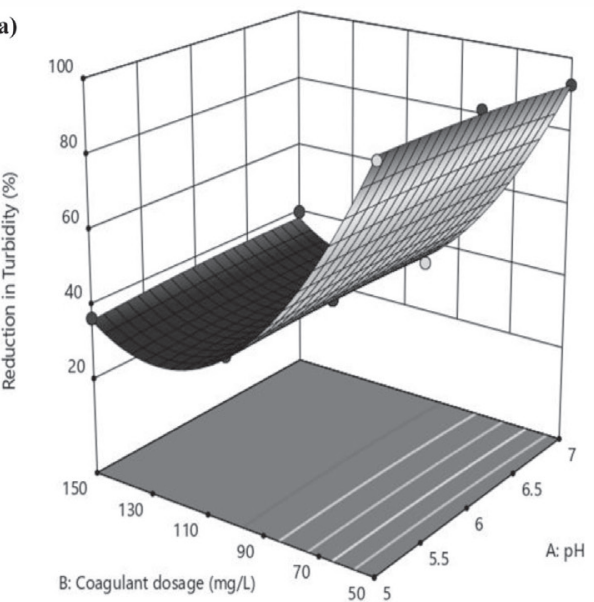

c)

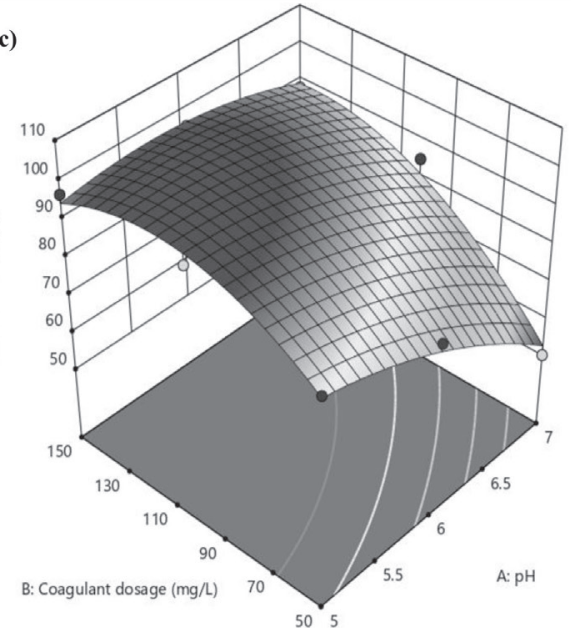

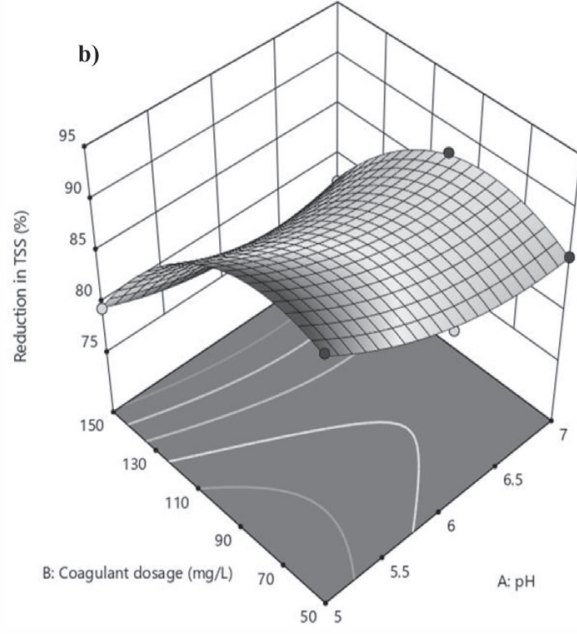

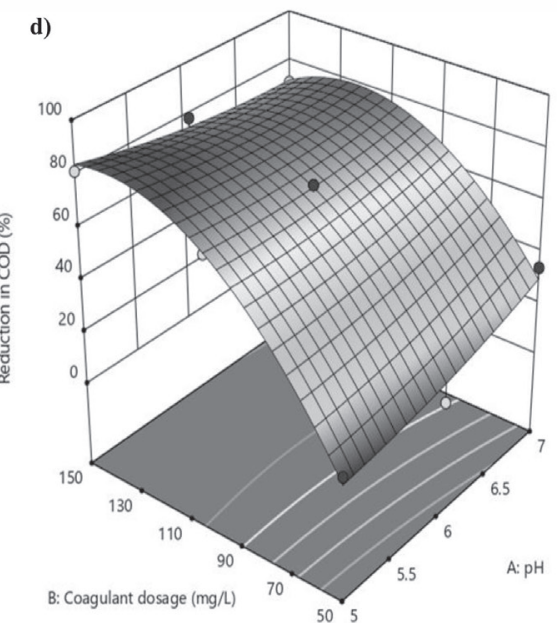

Fig. 1. Three dimensional response surface plots showing the effect of $\mathrm{pH}$ and coagulant dosage on reduction of a) Turbidity, b) TSS, c) BOD (d) COD by coagulation using watermelon (citrullus lanatus) coagulant.

hand, a dosage of $150 \mathrm{mg} / \mathrm{L}$ was also decreased in turbidity to 36.5 and TSS to $79.5 \%$. However, in Fig. 2(a-b), it is clear that at $\mathrm{pH} 5$ and coagulant dosage of $50 \mathrm{mg} / \mathrm{L}$, the removal of turbidity and TSS were only 41.8 and $64.4 \%$ respectively. When the coagulant dosage was increased to $100 \mathrm{mg} / \mathrm{L}$ and $150 \mathrm{mg} / \mathrm{L}$, the percentage removal of turbidity was reduced more to $(30.1$ and $32.1 \%)$ while TSS increased to $(88.9$ and $88.4 \%$ ) respectively. The natural coagulant is more effective in turbidity at lower $\mathrm{pH}$ and dosage. Thus, TSS was found to be efficient regardless of dosage and $\mathrm{pH}$.

The results of the experiments conducted for the effect of $\mathrm{pH}$ and dosage on the BOD and COD removal are presented in Fig. 1(c-d) and 2(c-d). The results from Fig. 1(c-d) showed that at $\mathrm{pH} 5$ and coagulant dosage of $50 \mathrm{mg} / \mathrm{L}$, the removal of BOD was $86.4 \%$ and COD removal was very low at $14.3 \%$. When coagulant dosage was increased to $100 \mathrm{mg} / \mathrm{L}$, the percentage removal of BOD and COD increased to 97.7 and $71.4 \%$ respectively. Again, after the coagulant dosage was increased to $150 \mathrm{mg} \mathrm{L}$, the removal were remained high of 96.6 for BOD and $81.4 \%$ for COD. Further, Fig. 2(c-d) resulted in lower BOD (44\%) and COD (22.9\%) in $50 \mathrm{mg} / \mathrm{L}$ at $\mathrm{pH}$ 5. When dosage increased to $100 \mathrm{mg} \mathrm{\textrm {L } ^ { - }}$ and $150 \mathrm{mg} \mathrm{L}$; the percentage removal of BOD increased to 88.6 and $92 \%$ respectively. Thus, as $\mathrm{pH}$ increases to 6 and 7 and dosage of 100 and $150 \mathrm{mg} / \mathrm{L}$ in both natural coagulants the percentage removal of BOD remains high. However, the percentage removal of COD remains low in $100 \mathrm{mg} / \mathrm{L}(11.4 \%)$ and $150 \mathrm{mg} / \mathrm{L}(48.6 \%)$. The study showed that the response surface method was very effective to obtain the optimum condition and maximizing the removal of turbidity, BOD and COD.

\section{FTIR Results from Watermelon (Citrullus lanatus) and Cantaloupe (Cucumis melo) Seeds}

The Fourier transform infrared spectroscopy (FTIR) technique was an important tool to identify some functional groups present in the natural coagulant of watermelon (C. lanatus) and cantaloupe (C. melo) as shown in Fig. 3. The spectrum shows the plot of absorption intensity versus the wavelength frequencies of the compound. Two similar strong peak patterns observed both in $C$. lanatus and $C$. melo at 

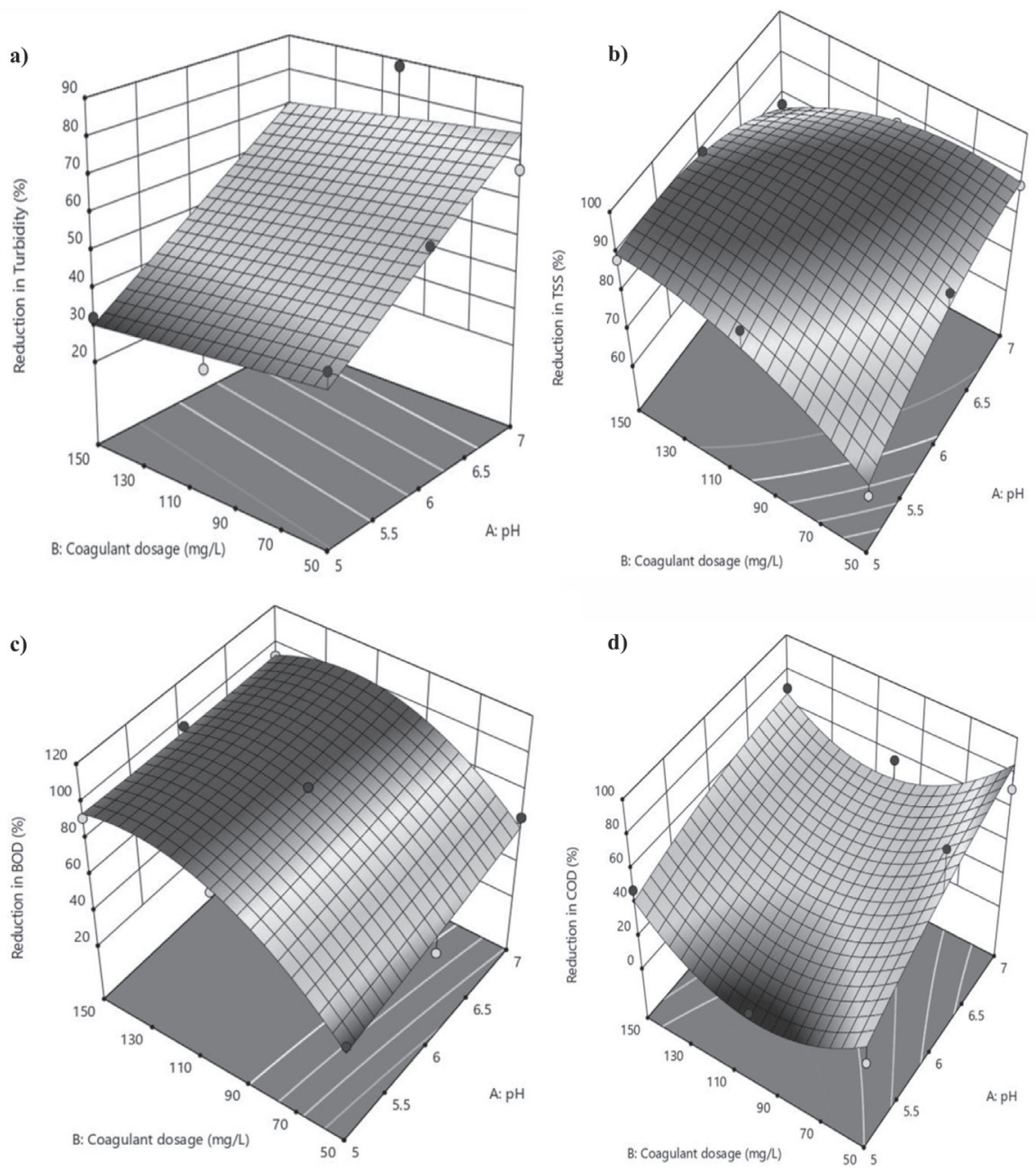

Fig. 2. Three dimensional response surface plots showing the effect of $\mathrm{pH}$ and coagulant dosage on reduction of a) Turbidity, b) TSS, c) BOD (d) COD by coagulation using cantaloupe (cucumsis melo) coagulant.

$3269.34 \mathrm{~cm}^{-1}$ which signify the presence of strong bands of $\mathrm{O}-\mathrm{H}$ bond that indicates the presence of water. In addition, stretching was observed in the region between 2500 and $3000 \mathrm{~cm}^{-1}$ indicates the presence of C-H bond. These two main functional groups found in $C$. lanatus and $C$. melo could be attributed to the contents of protein [47]. These results are in agreement obtained in the high percentage removal of turbidity in the study.

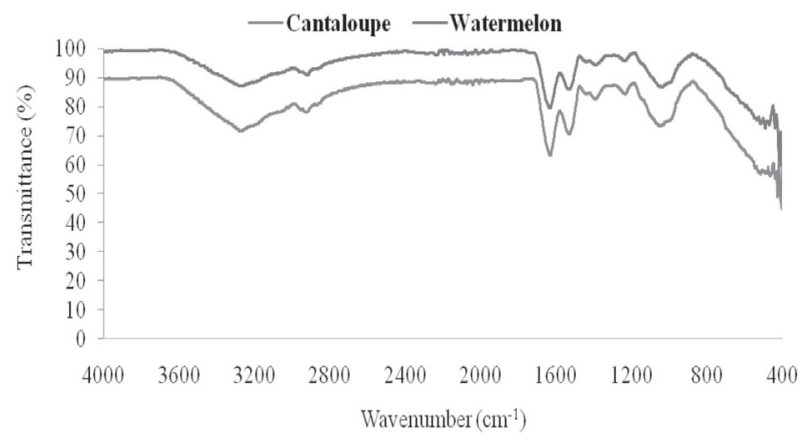

Fig. 3. FTIR spectra of extracting seeds from watermelon $(C$. lanatus) and cantaloupe (C. melo) seeds.
Thus, some studies show that natural coagulant from $M$. oleifera seeds have greater effectiveness for the removal of high turbidity in wastewater [48]. Moreover, the region between 1400 and $1700 \mathrm{~cm}^{-1}$ corresponds to the carbonyl, $\mathrm{C}=\mathrm{O}-\mathrm{NHR}$, amine, $\mathrm{NH}_{2}$ and ammonium, $\mathrm{NH}^{+3}$ bands. As it can be seen in the result strong bands similarity of $\mathrm{C}=\mathrm{O}$ stretching was observed in the region between 1500 and $1750 \mathrm{~cm}^{-1}$. Also, free amino acids aggravate the catalytic property, thus improving charge neutralization of suspended solids and bridging the flocculation [49]. One comparable bending band observed at 1000 and 1500 which verified the presence of amino group in both natural coagulants $(C$. lanatus and $C$. melo) which reduce the TSS and COD levels after the treatment [50]. Moreover, it was observed that the two plant species seeds obtained almost similar spectra.

\section{Conclusion}

Application and optimization of natural coagulants from watermelon (C. lanatus) and cantaloupe (C. melo) 
in the coagulation-flocculation process were investigated in this study. The response surface methodology design using CCD was applied to determine the operating conditions of turbidity, total suspended solids, BOD, and COD. Based on the results demonstrated, the removal efficiency of both natural coagulants was highly effective. The natural coagulants of $C$. lanatus and $C$. melo achieved the maximum treatment performance of TSS (92.8\% and 87.7\%) and BOD (92.1\% and 80.1\%) under the dosage of $72 \mathrm{mg} / \mathrm{L}$ and $76.7 \mathrm{mg} / \mathrm{L}$ in $\mathrm{pH}$ (5 and 7) respectively. Instead of discarding or simply thrown away the seeds, a possible option is to convert into a natural coagulant to purify water and wastewater. The ability of natural plant seeds from $C$. lanatus and $C$. melo to act as a natural coagulant for treatment of municipal wastewater can be a suitable and effective pre-treatment option instead of conventional coagulants.

\section{Acknowledgement}

The authors would like to acknowledge the support of Salalah Sanitary Drainage and Services (SSDS) and Salalah College of Technology (SCT), Engineering Department, Sultanate of Oman, for the opportunity to perform the various stages of research. Also, to the School of Chemical Engineering, Vellore Institute of Technology (VIT), India, for the guidance and evaluation of the research.

\section{Conflict of Interest}

The authors declare no conflict of interest.

\section{References}

1. YANG G., ZHANG G., WANG H. Current state of sludge production, management, treatment and disposal in China. Water Research, 78, 60, 2015.

2. MORRISON G., FATOKI O.S., PERSSON L., EKBERG A. Assessment of the impact of point source pollution from the Keiskammahoek Sewage Treatment Plant on the Keiskamma River-pH, electrical conductivity, oxygendemanding substance (COD) and nutrients. Water SA, 27 (4), 475, 2001.

3. JOAQUIN A.A., NIRMALA G. Statistical modeling and process optimization of coagulation-flocculation for treatment of municipal wastewater. Desalination and Water Treatment, 157, 90, 2019.

4. EDZWALD J.K. Rapid-Mixing Tanks for Coagulation: Are They Needed? Journal of the New England Water Works Association, 128 (1), 1, 2014.

5. SIVARANJANI S., RAKSHIT A. Study on removal efficiency of blended coagulants on different types of wastewater. Nature Environment and Pollution Technology 16 (1), 107, 2017.

6. NAIDOO S., OLANIRAN A.O. Treated wastewater effluent as a source of microbial pollution of surface water resources. International Journal of Environmental Research and Public Health, 11 (1), 249, 2014.

7. SPILLER M., VREEBURG J.H., LEUSBROCK I., ZEEMAN G. Flexible design in water and wastewater engineering - definitions, literature and decision guide. Journal of Environmental Management, 149, 271, 2015.

8. BINTI SAHARUDIN N.F.A., NITHYANANDAM R. Wastewater treatment by using natural coagulant. 2nd Eureca, 4, 213, 2013.

9. AGUNBIADE M.O., POHL C.H., ASHAFA A.O. A review of the application of biofloccualnts in wastewater treatment. Polish Journal of Environmental Studies, 25 (4). 1381, 2016.

10. GOLOB V., VINDER A., SIMONIČ M. Efficiency of the coagulation/flocculation method for the treatment of dyebath effluents. Dyes and Pigments, 67 (2), 93, 2005.

11. TEH C.Y., BUDIMAN P.M., SHAK K.P.Y., WU T.Y. Recent advancement of coagulation-flocculation and its application in wastewater treatment. Industrial \& Engineering Chemistry Research, 55 (16), 4363, 2016.

12. JOHNSON P.N., AMIRTHARAJAH A. Ferric chloride and alum as single and dual coagulants. Journal-American Water Works Association, 75 (5), 232, 1983.

13. MCCURDY K., CARLSON K., GREGORY D. Floc morphology and cyclic shearing recovery: comparison of alum and polyaluminum chloride coagulants. Water Research, 38 (2), 486, 2004.

14. DAUD Z., AZIZ A., LATIF A., MAO L. Coagulationflocculation in leachate treatment by using ferric chloride and alum as coagulant. International Journal of Engineering Research and Applications, 2 (4), 1929, 2012.

15. NG M., LIANA A.E., LIU S., LIM M., CHOW C.W.K, WANG D., DRIKAS M., AMAL, R. Preparation and characterisation of new-polyaluminum chloride-chitosan composite coagulant. Water Research, 46 (15), 4614, (2012).

16. WU C., WANG Y., GAO B., ZHAO Y., YUE Q. Coagulation performance and floc characteristics of aluminum sulfate using sodium alginate as coagulant aid for synthetic dying wastewater treatment. Separation and Purification Technology, 95, 180, 2012.

17. NDABIGENGESERE A., NARASIAH K.S. Use of Moringa oleifera seeds as a primary coagulant in wastewater treatment. Environmental Technology, 19 (8), 789, 1998.

18. BHUPTAWAT H., FOLKARD G.K., CHAUDHARI, S. Innovative physico-chemical treatment of wastewater incorporating Moringa oleifera seed coagulant. Journal of Hazardous Materials, 142 (1-2), 477, 2007

19. CAMACHO F.P., SOUSA V.S., BERGAMASCO R., TEIXEIRA M.R. The use of Moringa oleifera as a natural coagulant in surface water treatment. Chemical Engineering Journal, 313, 226, 2017.

20. ADINOLFI M., CORSARO M.M., LANZETTA R., PARRILlI M., FOLKARD G., GRANT W., SUTHERLAND J. Composition of the coagulant polysaccharide fraction from Strychnos potatorum seeds. Carbohydrate Research, 263 (1), 103, 1994.

21. RANI C.N., JADHAV, M.V. Enhancing filtrate quality of turbid water incorporating seeds of Strychnos potatorum, pads of Cactus opuntia and mucilage extracted from the fruits of Coccinia indica as coagulants. Journal of Environmental Research and Development, 7 (2), 668, 2012.

22. ABOULHASSAN M.A., SOUABI S., YAACOUBI A., BAUDU M. Coagulation efficacy of a tannin coagulant 
agent compared to metal salts for paint manufacturing wastewater treatment. Desalination and Water Treatment, 57 (41), 19199, 2016.

23. YIN C.Y. Emerging usage of plant-based coagulants for water and wastewater treatment. Process Biochemistry, 45 (9), 1437, 2010.

24. SIEVÄNEN K., KAVAKKA J., HIRSILÄ P., VAINIO P., KARISALMI K., FISKARI J., KILPELÄINEN I. Cationic cellulose betainate for wastewater treatment. Cellulose, 22 (3), 1861, 2015.

25. IMRAN Q., HANIF M.A., RIAZ M.S., NOUREEN S., ANSARI T.M., BHATTI H.N. Coagulation/flocculation of tannery wastewater using immobilized chemical coagulants. Journal of Applied Research and Technology, 10 (2), 79, 2012.

26. VISHALI S., ROSHINI S.K., SAMYUKTHA M.R. Towards zero waste production in the paint industry wastewater using an agro-based material in the treatment train. Environmental Monitoring and Assessment, 190 (10), 587, 2018.

27. VERMA A.K., DASH R.R., BHUNIA P. A review on chemical coagulation/flocculation technologies for removal of colour from textile wastewaters. Journal of Environmental Management, 93 (1), 154, 2012.

28. UGWU S.N., UMUOKORO A.F., ECHIEGU E.A., UGWUISHIWU B.O., ENWEREMADU C.C. Comparative study of the use of natural and artificial coagulants for the treatment of sullage (domestic wastewater). Cogent Engineering, 4 (1), 1365676, 2017.

29. VIJAYARAGHAVAN G., SIVAKUMAR T., KUMAR A.V. Application of plant based coagulants for waste water treatment. International Journal of Advanced Engineering Research and Studies, 1 (1), 88, 2011.

30. SATHISH S., VIKRAM S., SURAJ R. Effectiveness of turbidity removal from synthetic and tannery wastewater by using seeds of a natural coagulant Citrullus lanatus. Nature Environment and Pollution Technology, 17 (2), 551, 2018.

31. BANERJEE K., RAMESH S.T., GANDHIMATHI R., NIDHEESH P.V., BHARATHI, K.S. A novel agricultural waste adsorbent, watermelon shell for the removal of copper from aqueous solutions. Iranica Journal of Energy \& Environment, 3 (2), 143, 2012.

32. CRINI G. Recent developments in polysaccharide-based materials used as adsorbents in wastewater treatment. Progress in polymer science, 30 (1), 38, 2005.

33. KERMET-SAID H., MOULAI-MOSTEFA N. Optimization of turbidity and COD removal from pharmaceutical wastewater by electrocoagulation> Isotherm modeling and cost analysis. Polish Journal of Environmental Studies, 24 (3), 2015.

34. NAIR A.T., MAKWANA A.R., AHAMMED M.M. The use of response surface methodology for modelling and analysis of water and wastewater treatment processes: a review. Water Science and Technology, 69 (3), 464, 2014.

35. SAID K.A.M., AMIN M.A.M. Overview on the response surface methodology (RSM) in extraction processes. Journal of Applied Science \& Process Engineering, 2 (1), 2015.

36. MUHAMMAD I.M., ABDULSALAM S., ABDULKARIM A., BELLO, A.A. Water melon seed as a potential coagulant for water treatment. Global Journal of Research In Engineering, 15 (1), 17, 2015.

37. RAMAVANDI B. Treatment of water turbidity and bacteria by using a coagulant extracted from Plantago ovata. Water Resources and Industry, 6, 36, 2014.
38. SIVAMANI S., BASKAR R. Process design and optimization of bioethanol production from cassava bagasse using statistical design and genetic algorithm. Preparative Biochemistry and Biotechnology, 48 (9), 834, 2018.

39. MINER G. Standard methods for the examination of water and wastewater. American Water Works Association. Journal, 98 (1), 130, 2006.

40. NWABUEZE, T.U. Basic steps in adapting response surface methodology as mathematical modelling for bioprocess optimisation in the food systems. International Journal of Food Science \& Technology, 45 (9), 1768, 2010.

41. DOS SANTOS J.D., VEIT, M.T., PALÁCIO, S.M., GONCALVES G.D.C., FAGUNDES-KLEN M.R. Evaluation of the combined process of coagulation/ flocculation and microfiltration of cassava starch wastewater: Removal efficiency and membrane fouling. Water, Air, \& Soil Pollution, 228, 238, 2017.

42. BAAWAIN M.S., AL-FUTAISI A.M., AL-OMAIRI A., AL-JABRI M. Investigating municipal wastewater treatment in the sultanate of Oman. WIT Transactions on Ecology and the Environment, 145, 711, 2011.

43. ALMEIDA I.L., DE ASSUNCAO R.M., TARLEY C.R., SEGATELLI M.G., COELHO N.M.M. Characterization and use of Moringa oleifera seeds as biosorbent for removing metal ions from aqueous effluents. 62 (9), 2198, 2010.

44. MATEUS G.A.P., FORMENTINI-SCHMITT D.M., NISHI L., FAGUNDES-KLEN M.R., GOMES R.G., BERGAMASCO R. Coagulation/flocculation with Moringa oleifera and membrane filtration for dairy wastewater treatment. Water, Air, \& Soil Pollution, 228 (9), 342, 2017.

45. ADNAN O., ABIDIN Z.Z., IDRIS A., KAMARUDIN S., AL-QUBAISI M.S. A novel biocoagulant agent from mushroom chitosan as water and wastewater therapy. Environmental Science and Pollution Research, 24 (24), 20104, 2017.

46. SHAK K.P.Y., WU, T.Y. Coagulation-flocculation treatment of high-strength agro-industrial wastewater using natural Cassia obtusifolia seed gum: Treatment efficiencies and flocs characterization. Chemical Engineering Journal, 256, 293, 2014.

47. AHMAD A.L., WONG S.S., TENG T.T., ZUHAIRI A. Optimization of coagulation-flocculation process for pulp and paper mill effluent by response surface methodological analysis. Journal of Hazardous Materials, 145 (1-2), 162, 2007.

48. SRIDHAR R., SIVAKUMAR V., MARAN J.P., THIRUGNANASAMBANDHAM, K. Influence of operating parameters on treatment of egg processing effluent by electrocoagulation process. International Journal of Environmental Science and Technology, 11 (6), 1619, 2014.

49. BASKAN M.B., PALA, A. A statistical experiment design approach for arsenic removal by coagulation process using aluminum sulfate. Desalination, 254 (1-3), 42, 2010.

50. MUHAMAD M.H., ABDULLAH S.R.S., MOHAMAD A.B., RAHMAN R.A., KADHUM, A.A.H. Application of response surface methodology (RSM) for optimisation of COD, $\mathrm{NH}_{3-} \mathrm{N}$ and 2,4-DCP removal from recycled paper wastewater in a pilot-scale granular activated carbon sequencing batch biofilm reactor (GAC-SBBR). Journal of Environmental Management, 121, 179, 2013. 
